\title{
ASTRONOMY IN THE FORMER SOVIET UNION
}

\author{
NIKOLAI G. BOCHKAREV \\ Euro-Asian Astronomical Society \\ Universitetskij prospect 13, 119899, Moscow, Russia. \\ e-mail: boch@astronomy.msk.su
}

The Soviet Union developed science (including astronomy) to a high level, although largely isolated from the rest of the world. In 1990 about $2 \%$ of GDP, i.e. tenths of a billion dollars per year, were invested in science. The USSR occupied fourth place in the world in respect of its investment in science. More than a million persons worked in scientific research, with about 3,000 of those in astronomy.

During the Soviet era a strong technical base was created: the 6-meter telescope at the SAO (North Caucasus), two telescopes with mirrors of 2.6 meters diameter (Crimean and Byurakan Observatories), the 2-meter telescope at Shemakha Observatory and a large number of smaller telescopes. Specialized solar telescopes and observatories were spread over all longitudes, reaching to the Pacific; many radio-telescopes, including the largest of its class such as RATAN-600 at SAO and URAN-4 (Ukraine) for the decameter range, the telescopes of Puschino Radio-astronomical observatory and many others. Besides these, there were the Cherenkov high-energy gamma-rays detector; neutrino telescopes at Bakasan Observatory (North Caucasus) and in the depth of Baikal Lake; space-based observatories were at work, space probes were being launched towards bodies in the Solar System.

At sites with good astroclimate: at the Central Asia mountains and Kazakhstan (Maidanak Mountain in Uzbekistan, Sanglok Mountain in Tajikstan, Dushak-Egekdag Mountain in Turkmenistan, Tien-Shan Mountains) new astronomical observatories were built and equipped with small but quite modern telescopes. Large astronomical projects were carried out: VLBI-system "Quasar", steerable 70-meter dish radiotelescope RT-70 for the millimeter range, and so on. A gravitational-wave antenna of moderate size was under construction and a large space astronomical project was being developed.

On the other hand, the USSR was well behind the Western World in equipping these instruments with light receivers and computers and in the development of communications.

The principal instruments were concentrated in institutions and observatories of the Academy of Science of the USSR and of Academies of the republics. Moreover, many of the republics had also their own observatories. The professionals were educated at the universities both of Russia and of a number of other republics (Ukraine, Armenia, Georgia, Uzbekistan).

The Astronomical-Geodesic Society was the unique social scientific organization uniting astronomers. The fraction of professional astronomers constituted $1 \%$ of the full number of the Society members and therefore their problems could not be solved through that organization. This was a reason for the organization of a professional astronomical society -the Soviet Astronomical Society (now an international non-profit society, the Euro-Asian Astronomical Society or EAAS) in the year 1990 .

After the USSR broke down, industrial production declined rapidly (especially since 1992). Thus the GDP in Russia was approximately halved. The share of GDP invested in science went down to less than $0.5 \%$-a decline to about one tenth in absolute value. The prestige of science has decreased too. The number of persons working in science has halved, and the influx of young people into science has fallen abruptly. Scientists' salaries are smaller than average salaries, being quite near the subsistence wage. Similar things have happened in other former Soviet republics.

The situation in the Baltic countries is a little better, although professional attestation resulted in a reduction of personnel by more than half. However, salaries there at present are about $\$ 200$ $\$ 300$ a month and are paid regularly. The principal institutions have access to the Internet. Russian 
forces left behind in Venspils, in Latvia, a 32-meter and a 16-meter dish. Latvian astronomers, in collaboration with Swedes and Russians, have recently obtained their first observational results with these dishes.

In Russia, a scientist's average salary is about $\$ 100$ a month; moreover, in 1996, there were considerable delays in payment. Some people spontaneously left, but the main body of highly qualified specialists has been conserved. There is a number of grants for scientific research from both Russian and non-Russian sources, but small university observatories dedicated to the professional training of young astronomers are in danger.

More than half of all the astronomers of the former Soviet Union live in Russia although the predominant number of optical observatories are at present in NIS countries. For political reasons, Russian astronomers find it difficult to use these observatories. SAO of the Russian Academy of Sciences (North Caucasus) is the principal observational station in Russia. All the instruments (RATAN-600, 6-meter, 1-meter and 0.6-meter telescopes) are in working order and, as a national observatory, serve astronomers from all over the former Soviet Union. The telescopes have been equipped with modern CCD cameras.

Observational bases in Kazakhstan and Uzbekistan formerly belonging to the Sternberg Astronomical Institute of Moscow University have been nationalized, and Russian astronomers can use them only by agreement. The Pulkovo Observatory stations in the southern hemisphere (Chile and Bolivia) cannot be used; the Shorbulak Station in the Pamirs has been nationalized and that at Nakhichevan is idle. The fate of the station under construction in Armenia is unknown. No-one has visited these stations for a long time. The Armenian observational station of St Petersburg University is also laid up.

Fortunately, Puschino radio-astronomical Observatory, solar observatories in Siberia (near Irkutsk and in Ussukijsk, near the Pacific), and a small optical observatory which belongs to the Ural University are working. The Russian part of VLBI "Quasar" is being completed, step by step. A number of steerable dishes near Moscow are being tested. Recently, the dominant part of Russian astronomical institutions were connected to Internet (see poster to JD 12), but there are some institutions that do not have even e-mail.

The situation in Ukraine matches that in Russia: in 1995 salaries were raised to $\$ 100 \mathrm{a}$ month, but are paid far less regularly than even in Russia. The Crimean Astrophysical Observatory belongs to the Ministry of Science of Ukraine. All the telescopes work. Other observatories work too.. At Nikolaev Astronomical Observatory a new remote controlled meridian circle has been commissioned recently. Several Kiev and Crimean Institutions are connected to the Internet. The Terksol Peak (North Caucasus) Observatory, originally built by the Academy of Science of the Ukraine, has become Ukraine-Russian joint property. A 2-meter Zeiss telescope has been installed there; testing observations are about to be carried out. Another such telescope of Odessa Observatory on DushakEregdag Mountain in Turkmenistan is used for several months a year.

The salaries in the rest of the FSU countries are usually about $\$ 20-\$ 40$ a month, but are paid regularly or with only a small delay. The sum is near to or a bit lower than the living wage. There is no governmental grants system; a part of working groups gets international grants, in rare cases, in collaborating with Russia. The main staff keeps working.

The situation in Transcaucasus countries has been far harder because of the war. In Georgia and in Armenia power supply was available for just several hours a day. During the last 1.5 year, however, the situation has improved and become stable. Power supply is available for 12-14 hours a day all over the countries, and practically all the day through in the capitals. There is limited access to the Internet, astronomical observatories have e-mail.

The 2.6-meter telescope of Byurakan Observatory is working and has recently been equipped with a modern CCD camera (through a collaborative program with France) but the 1-m Schmidt telescope is not functioning. All the other optical telescopes are in working order, although the the radio-optical telescope $(\mathrm{D}=32 \mathrm{~m})$ needs adjustment and has no modern light receptors. The Armenian Institute for Space Astrophysics works.

All the instruments of the Abastumani Observatory of the Academy of Science of Georgia are in working order, but the main $1.25-\mathrm{m}$ solar telescope needs repair. Monitoring of the Sun has begun. Observational programs in collaboration with Russia are being carried out. Nevertheless, because the central heating is out of order, the largest part of the staff works mainly in the Tbilsi Laboratory of the Observatory. 
Up to now there are no contacts with Azerbaijan astronomers, not even by e-mail. The 2-m telescope of Shemakha Observatory is not working because of damage to its mechanical parts. Power supply used to be irregular because the power lines were in disrepair. The Batabad station of Shemakha Observatory in Nakhichevan was destroyed during the war.

The economical situation in the Central Asian countries and Kazakhstan is difficult but all astronomical institutions are at work. All the city administrative institutions have e-mail. The Internet is accessible mainly on commercial terms -an opportunity that cannot be used by astronomers for financial reasons. Highland observational stations usually lack even telephonic communication.

The hardest situation is that in Tajikistan. During 1994 no salaries were paid. Sangkok Observatory is not in use, but the 1- m telescope is in working order. Guissar Observatory continues working.

The Maidanak Observatory in Uzbekistan was built by four republics of the former Soviet Union. In 1993 it was nationalized and is now the property of the Astronomical Institute of the Academy of Science of Uzbekistan. Instruments constructed by Lithuania and Moscow (SAI) are in cooperative use. A $1.5-\mathrm{m}$ telescope constructed by the SAI has been out of work. Astroclimatic testings carried out with ESO equipment have confirmed very good seeing at the site. At the Kumbel station of the Astronomical Institute of Uzbekistan observations in the frame of international helioseismological programs are carried out actively. Kitab latitude station is beginning to re-equip in order to be incorporated into international programs. The construction of the radiotelescope RT-70 on Suffa Plateau has been temporarily stopped: to cover the high cost of this telescope, Russian-Uzbek agreement is needed at governmental level, but has not yet been signed.

The Astrophysical Institute of the Academy of Science of Kazakhstan has nationalized the SAI Highland Tien-Shan Station. The laboratory building of the station is leased, the telescopes are used cooperatively. One of the two 1-m telescopes has not been put to work. Assy-Turgen Plate and other equipment is used permanently.

Solar telescopes near Vanovski Village in Turkmenistan are at work. Construction at DushakEregdag Mountain is slowly progressing, telescopes which are already in working order are being used, but not intensively.

Libraries all over the former Soviet Union have difficulties in acquiring periodicals. In a number of cases library programs of societies and foundations (AAS, AAAS, ISF) provided great help in acquiring English-language literature. The AAS award of a three-year subscription to the electronic version of the ApJ for astronomical institutions of FSU countries should also be mentioned. Books and Symposia Proceedings are almost inaccessible. Because of difficulties in acquiring Russianlanguage periodicals in astronomy, the EAAS initiated about 2.5 years ago a library program of its own, with the aim of providing subscriptions to Astron. Zh., Pisma v Astron. Zh. and other popular-science magazines, as well as to the English-language journal of the EAAS, Astronomical and Astrophysical Transactions.

Apart from the EAAS, national astronomical societies have been created later in a number of FSU countries. The Astronomical Association of the Ukraine unites collectives of astronomers (astronomical institutions) and a number of individual astronomers. Societies in the Baltic countries unite professional and amateur astronomers. Tajik and Odessa (Ukraine) astronomical societies, uniting professional astronomers and teachers of astronomy at pedagogical institutes, are affiliated members of EAAS.

The EAAS unites about 750 professional astronomers from 31 countries, including all the FSU countries with well developed astronomy, as well as 31 collective members, 9 associations (those of teachers of astronomy at pedagogical institutes, teachers of astronomy at secondary schools, planetaria, amateur astronomers associations). The EAAS supports Olympics in astronomy for schoor students, awards prizes, actively collaborates with governmental institutions and astronomical societies of other countries on problems of support and development of astronomy. 